\title{
Effects of Bi doping on dielectric and ferroelectric properties of PLBZT ferroelectric thin films synthesized by sol-gel processing
}

\author{
HUA WANG ${ }^{\dagger, \downarrow, *}$, LI LIU ${ }^{\dagger}$, JI-WEN XU ${ }^{\dagger, \ddagger}$, CHANG-LAI YUAN ${ }^{\dagger,+}$ and LING YANG ${ }^{\dagger}$ \\ ${ }^{\dagger}$ School of Materials Science and Engineering, ${ }^{\ddagger}$ Guangxi Key Laboratory of Information Materials, Guilin University \\ of Electronic Technology, Guilin 541 004, China
}

MS received 21 November 2011; revised 31 July 2012

\begin{abstract}
Pb}_{0.95}\left(\mathrm{La}_{1-y} \mathrm{Bi}_{y}\right)_{0.05}\right]\left[\mathrm{Zr}_{0.53} \mathrm{Ti}_{0.47}\right] \mathrm{O}_{3}$ (PLBZT) ferroelectric thin films have been synthesized on indium tin oxide (ITO)-coated glass by sol-gel processing. PLBZT thin films were annealed at a relatively low temperature of $550^{\circ} \mathrm{C}$ in oxygen ambient. Effects of $\mathrm{Bi}$ doping on structure, dielectric and ferroelectric properties of PLBZT were investigated. Bi doping is useful in crystallization of PLBZT films and promoting grain growth. When the Bi-doping content $y$ is not more than $\mathbf{0 . 4}$, an obvious improvement in dielectric properties and leakage current of PLBZT was confirmed. However, when the Bi-doping content is more than $\mathbf{0 \cdot 6}$, the pyrochlore phase appears and the remnant polarization $P_{\mathrm{r}}$ of PLBZT thin films is smaller than that of $\left(\mathrm{Pb}_{1-x} \mathrm{La}_{x}\right)\left(\mathrm{Zr}_{1-y} \mathrm{Ti}_{\mathrm{y}}\right) \mathrm{O}_{3}$ (PLZT) thin films without Bi doping. PLBZT thin films with excessive Bi-doping content are easier to fatigue than PLZT thin films.
\end{abstract}

Keywords. Ferroelectric thin film; $\left(\mathrm{Pb}_{1-x} \mathrm{La}_{x}\right)\left(\mathrm{Zr}_{1-y} \mathrm{Ti}_{y}\right) \mathrm{O}_{3}$ (PLBZT); Bi doping; sol-gel.

\section{Introduction}

Non-volatile memories occupy an increased share of the growing memory market and becoming an indispensable component of memory circuits (Scott 2005; Tang et al 2007). $\left(\mathrm{Pb}_{1-x} \mathrm{La}_{x}\right)\left(\mathrm{Zr}_{1-y} \mathrm{Ti}_{y}\right) \mathrm{O}_{3}$ (PLZT) thin films are attracting much attention for their ferroelectric and optical applications such as ferroelectric random access memories, infrared sensors and electro-optic devices, due to their high optical transparency, outstanding ferroelectric and electro-optic properties (Gaidi et al 2004; Khodorov and Gomes 2006; Leclerc et al 2006; Singh et al 2008). However, PLZT thin films have a serious drawback i.e. they have low polarization retention and large leakage current, which enormously restrict their applications. Much work has been done in the past to study the effects of substitution in the $\mathrm{Pb}$ site of PZT, but not much work has been done to report the effect of double doping at the $\mathrm{Pb}$ site. The $\mathrm{La}-\mathrm{K}$ double doping PZT (PLKZT) ceramics show that their transition temperature and related parameters of ferroelectric phase are slowly influenced by the double doping of $\mathrm{La}$ and $\mathrm{K}$ at the $\mathrm{Pb}$ site (Mal and Choudhary 1997). La-Cs double doping PZT (PLCZT) ceramics can undergo diffuse phase transition with higher Cs concentration and the pairs of doping at the $\mathrm{Pb}$ sites created more structural disorder in PZT (Choudhary and Mal 2002). Goel et al (2004) and
Goel and Yadav (2007) have reported that the La-Bi double doping PZT (PLBZT) ceramics have a significant effect on the dielectric properties of PZT system. Shannigrahi et al $(1999,2002)$ reported that the La-Li double doping PZT (PLLZT) thin films sol-gel-grown on $\mathrm{Pt} / \mathrm{Ti} / \mathrm{SiO}_{2} / \mathrm{Si}$ substrates exhibited fatigue-free behaviour up to $6.5 \times 10^{10}$ switching cycles and have high dielectric constant of $10^{4}$ and remnant polarization of $14-24 \mu \mathrm{C} / \mathrm{cm}^{2}$.

Ferroelectric thin films were usually deposited on Pt/Ti/ $\mathrm{SiO}_{2} / \mathrm{Si}$ substrates. For the optical-electrical application, substrate-coated transparent oxide electrodes are needed. Glass substrate coated indium tin oxide (ITO) is a good choice, since it has been widely applied in manufacture of transparent conductors and depositing PLZT ferroelectric films (Zheng et al 2005; Yoon et al 2008; Pak et al 2010). High annealing temperature is another problem in PLZT thin films synthesized on ITO-coated glass because common glass softens at high temperature. However, previous studies reported that annealing temperature higher than $600{ }^{\circ} \mathrm{C}$ is required to synthesize PLZT thin films (Khodorov and Gomes 2006; Singh et al 2008; Yoon et al 2008).

In this paper, synthesis and characterization of Bi-doping PLBZT thin films on ITO-coated glass substrates through sol-gel process is described. Effects of $\mathrm{Bi}$ doping on structure, dielectric and ferroelectric properties have been investigated.

\section{Experimental}

Prior to being coated, ITO-coated glass substrates were ultrasonically cleaned in distilled water, acetone and methanol.

\footnotetext{
*Author for correspondence (wh65 @ tom.com)
} 
Lead acetate (excess 10\% to compensate the lead loss during annealing process), zirconium nitrate, lanthanum nitrate and bismuth nitrate were dissolved, respectively, in 2-methoxyethanol at $80{ }^{\circ} \mathrm{C}$ and dehydrated at $120{ }^{\circ} \mathrm{C}$. Then, tetra-butyl titanate was dissolved in acetylacetone and 2-methoxyethanol at $40{ }^{\circ} \mathrm{C}$. The spin-on technique was employed to deposit solution on ITO-coated glass substrates. After spinning onto substrates, wet films were kept on a hot plate in air at $300^{\circ} \mathrm{C}$ for $10 \mathrm{~min}$ to remove solvents and other organics. The desired thickness of PLBZT thin films was achieved by multiple spin-bake processes. The dry multilayer films were annealed for $30 \mathrm{~min}$ at $550{ }^{\circ} \mathrm{C}$ in oxygen ambient.

For determination of electrical properties, capacitors were formed by sputtering platinum $(\mathrm{Pt})$ electrodes of $0.1 \mathrm{~mm}$ diameter through a shadow mask on PLBZT films. The phase structure of films was analysed by X-ray diffraction (XRD), ferroelectric behaviour was determined using ferroelectric tester (Radiant P-WS), dielectric properties of films were measured using an impedance analyser (Agilent 4294A) and leakage current measurements were performed using source meter (Keithley 4200).

\section{Results and discussion}

Figure 1 shows XRD patterns of $\left[\mathrm{Pb}_{0.95}\left(\mathrm{La}_{1-y} \mathrm{Bi}_{y}\right)_{0.05}\right]$ $\left[\mathrm{Zr}_{0.53} \mathrm{Ti}_{0.47}\right] \mathrm{O}_{3}$ (PLBZT, $y=0.2,0.4,0.6$ and 0.8 ) and PLZT $(y=0.0)$ thin films. It can be seen that the locations of the peaks in XRD patterns of all samples are coincident, which indicate that there are no distinct differences in phase structure of PLZT and PLBZT thin films with different Bi-doping contents. When the Bi-doping content is not more than 0.6, PLBZT films are wellcrystallized and exhibit a polycrystalline perovskite phase structure, with no pyrochlore phase, which is similar to the

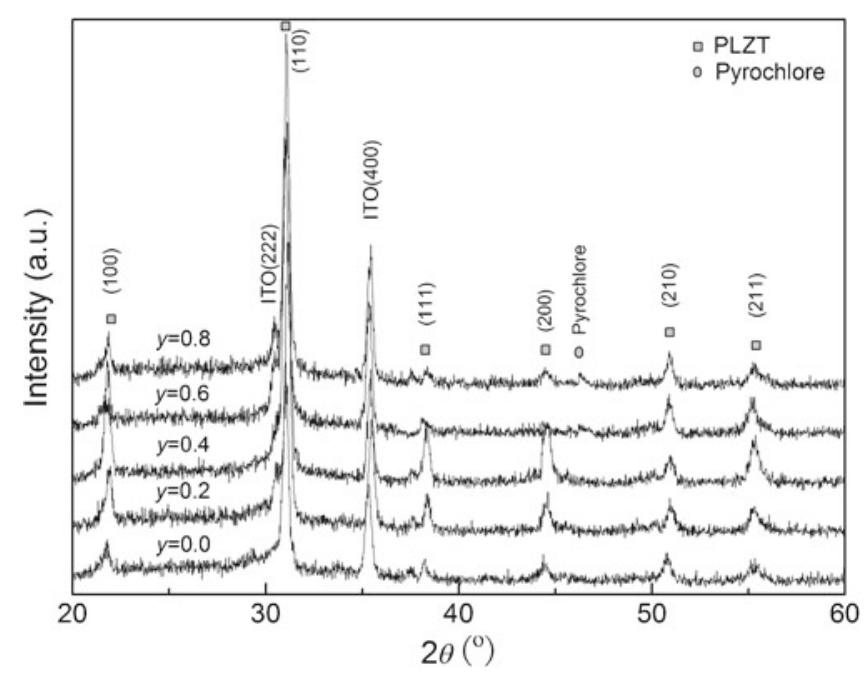

Figure 1. XRD patterns of $\left[\mathrm{Pb}_{0.95}\left(\mathrm{La}_{1-y} \mathrm{Bi}_{y}\right)_{0.05}\right]\left[\mathrm{Zr}_{0.53} \mathrm{Ti}_{0.47}\right] \mathrm{O}_{3}$ thin films. phenomenon from earlier research on PLBZT ceramics by Goel et al (2004). On increasing Bi-doping content from $0 \cdot 1$ to 0.4 , reflection peaks become sharp and reflection intensities also increase, which indicate that Bi doping is useful in the crystallization of PLBZT films and promoting grain growth. But then, some reflection peaks such as (111) and (200) are suppressed when the Bi-doping content is more than 0.4 , which may be due to the structural disorder derived from the $\mathrm{Bi}^{3+}$ entering the $\mathrm{B}$-site to replace $\mathrm{Ti}^{4+}$ or $\mathrm{Zr}^{4+}$. Furthermore, when the Bi-doping content is more than $0 \cdot 6$, the peaks of the pyrochlore phase appear. In addition, a slight shift of characteristic peaks in XRD patterns of PLBZT films is observed, this suggests that La ions in the lattice were partly replaced by smaller Bi ions and then decrease the lattice constant.

The surface morphology and cross-section SEM photographs of PLBZT thin films with various Bi-doping contents are shown in figure 2. Dense, smooth and crack-free surface morphologies can be observed in all the films. With the increase in Bi-doping content from 0.2 to 0.6 , average grain size was obviously increased from about $50 \mathrm{~nm}$ in figure 2(a) to $100 \mathrm{~nm}$ in figure 2(c). Besides, it can also be seen that there is a clear boundary between PLBZT film and ITO-coated glass substrate in figure 2(d), which means that the interaction and interdiffusion between PLBZT films and ITO-coated glass substrates is inconspicuous.

Figure 3 shows polarization-electric field $(P-E)$ hysteresis curves of PLZT and PLBZT (Bi-doping content, $y=0 \cdot 6$ ) thin films, measurement with the structure of Pt/PLZT/ITO and Pt/PLBZT/ITO, respectively. As shown in figure 3, well-saturated hysteresis loops and almost the same values of coercive field, $E_{\mathrm{c}}$, are observed in PLZT $(y=0)$ and PLBZT $(y=0.6)$ thin films, but the remnant polarization, $P_{\mathrm{r}}$ value in PLBZT $(y=0 \cdot 6)$ thin films is smaller than that of PLZT $(y=0)$ thin films. Furthermore, it can be seen that the $P-E$ curves do not show any noticeable asymmetric behaviour resulting in imprint failures. Figure 4 is the remnant polarization, $P_{\mathrm{r}}$ and coercive field, $E_{\mathrm{c}}$ of PLBZT thin films as a function of Bi-doping content. From figure 4, it can be seen that the $P_{\mathrm{r}}$ and $E_{\mathrm{c}}$ of PLBZT thin films are strongly dependent on the Bi content, $y$. The remnant polarization, $P_{\mathrm{r}}$, retains almost the same value of $25 \mu \mathrm{C} / \mathrm{cm}^{2}$ with increase in Bi-doping content from 0.2 to 0.4 , then decreased drastically with the increasing Bi-doping content over 0.6. Meanwhile, the coercive field, $E_{\mathrm{c}}$, decreased slowly with the increase in Bi-doping content from 0.2 to 0.4 , then increased slightly. The radius of $\mathrm{Bi}^{3+}(0.103 \mathrm{~nm})$ is close to that of $\mathrm{La}^{3+}(0 \cdot 106 \mathrm{~nm})$ and it is quite probable that the $\mathrm{Bi}$ ions enter La ions simultaneously, thereby, the strength of spontaneous polarization does not change obviously. However, the radius of $\mathrm{Bi}^{3+}$ is much closer to that of $\mathrm{Ti}^{4+}(0.0605 \mathrm{~nm})$ or $\mathrm{Zr}^{4+}(0.072 \mathrm{~nm})$ and can also replace the B-site in perovskite PZT lattice confirmed by Goel et al (2004). With the increase in Bi-doping content, some $\mathrm{Bi}^{3+}$ enter the B-site to replace $\mathrm{Ti}^{4+}$ or $\mathrm{Zr}^{4+}$, the substitution disorder in the arrangement of cations in one or more crystallographic sites of the structure results in increase in domain 

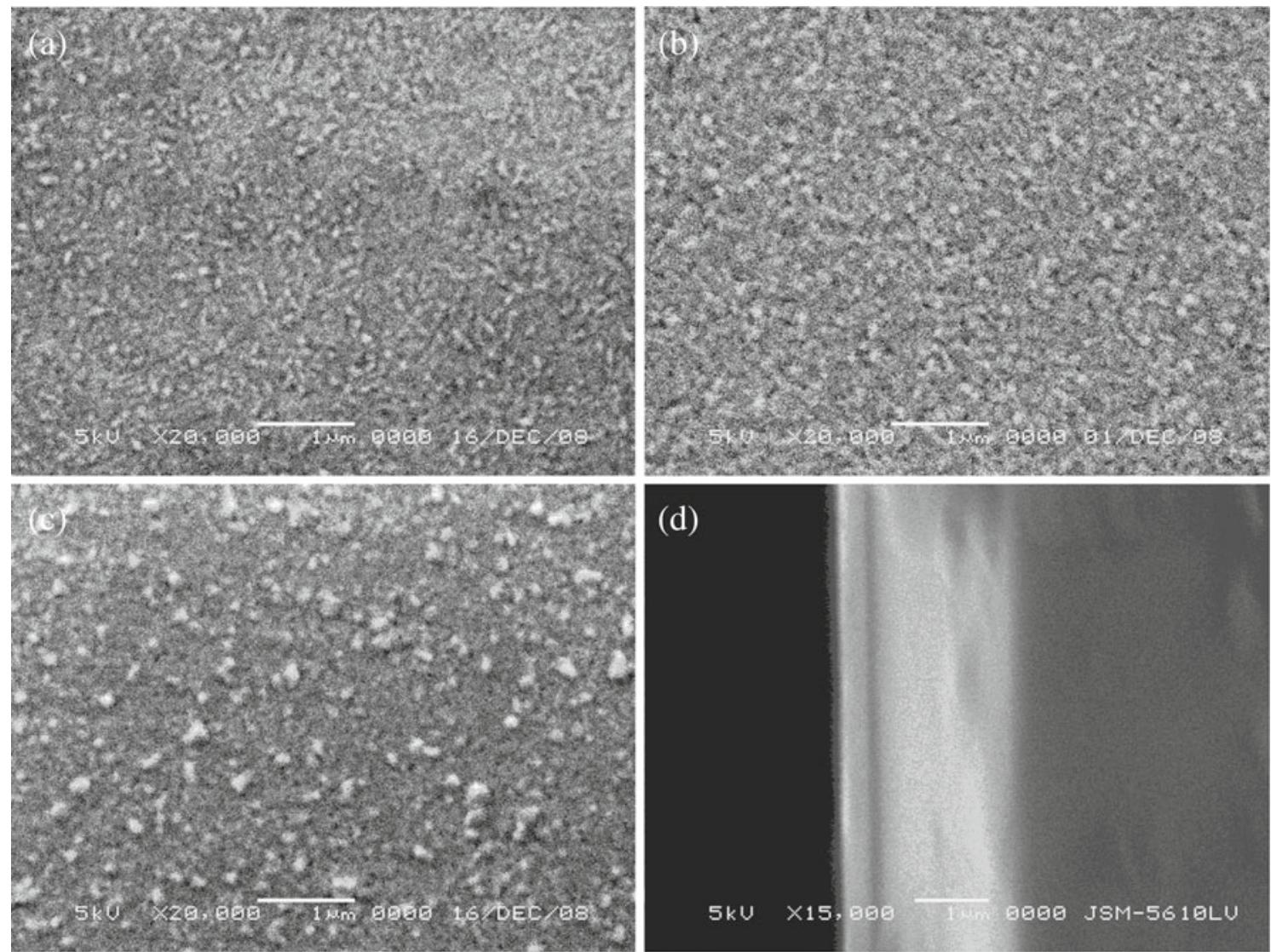

Figure 2. SEM images of $\left[\mathrm{Pb}_{0.95}\left(\mathrm{La}_{1-y} \mathrm{Bi}_{y}\right)_{0.05}\right]\left[\mathrm{Zr}_{0.53} \mathrm{Ti}_{0.47}\right] \mathrm{O}_{3}$ thin films with various Bi-doping contents, $y$. (a) $y=0 \cdot 2$, (b) $y=0 \cdot 4$, (c) $y=0.6$ and (d) cross-sectional morphology.

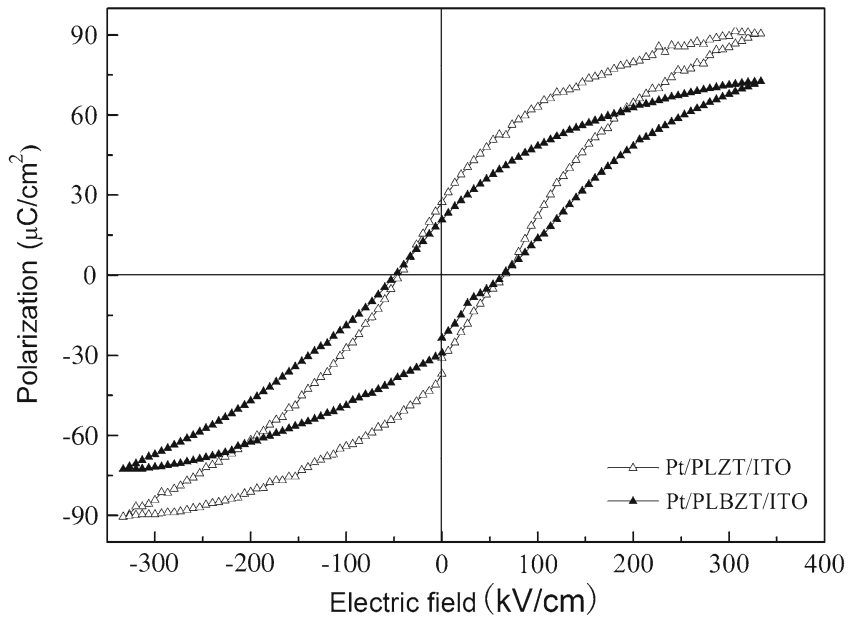

Figure 3. $P-E$ hysteresis curves of PLZT and PLBZT (Bidoping content, $y=0.6$ ) thin films.

wall mobility, thereby, both the strength of polarization and coercive field decrease.

The polarization decays of PLZT and PLBZT thin films were studied from the structures of Pt/PLZT/ITO and Pt/PLBZT/ITO (Bi-doping content, $y=0 \cdot 6$ ), respectively

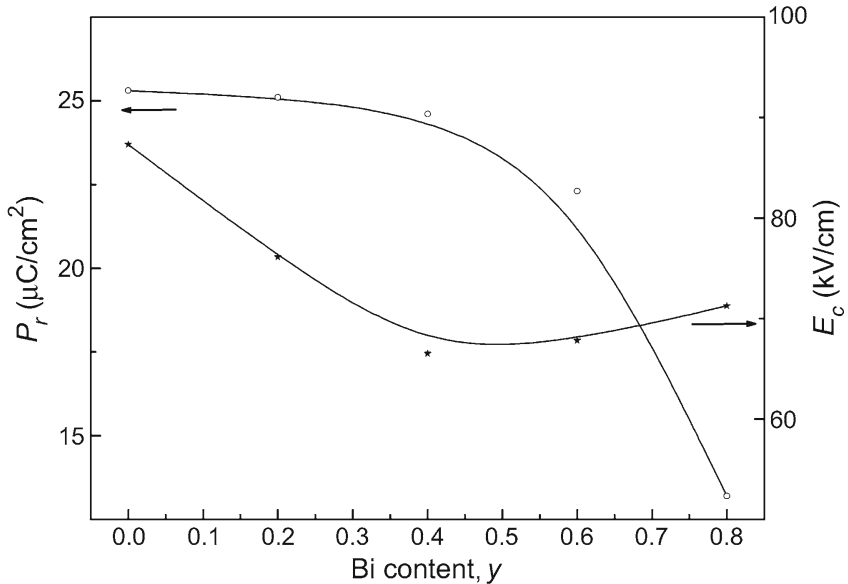

Figure 4. $P_{\mathrm{r}}$ and $E_{\mathrm{c}}$ of $\left[\mathrm{Pb}_{0.95}\left(\mathrm{La}_{1-y} \mathrm{Bi}_{y}\right)_{0.05}\right]\left[\mathrm{Zr}_{0.53} \mathrm{Ti}_{0.47}\right] \mathrm{O}_{3}$ thin films with various Bi contents, $y$.

by applying $10 \mathrm{kHz}$ bipolar pulses of $5 \mathrm{~V}$ amplitude. Figure 5 represents normalized polarizations of PLZT and PLBZT thin films as a function of switching cycles. In both the cases, films exhibit an identical trend in fatigue behaviour. In the initial long period (up to about $10^{8}$ cycles for PLZT and $10^{7}$ cycles for PLBZT), the $P_{\mathrm{r}}$ is almost constant, which 
is then followed by a final decay period. Even after $10^{9}$ switching cycles, the decay in $P_{\mathrm{r}}$ of PLZT is only $10 \%$ of the initial value; on the other hand, the same decay of $P_{\mathrm{r}}$ occurs in PLBZT thin films only after $10^{8}$ switching cycles. These suggest that the PLBZT thin films with excessive Bi-doping content are easier to fatigue than PLZT thin films. Compared with the fatigue characteristics of the $\mathrm{Pt} / \mathrm{Pb}_{0.98}\left(\mathrm{La}_{1-x} \mathrm{Li}_{x}\right)_{0.02}\left(\mathrm{Zr}_{0.55} \mathrm{Ti}_{0.45}\right) \mathrm{O}_{3} / \mathrm{Pt} \quad$ (Pt/PLLZT/Pt) capacitor (Shannigrahi et al 2002), this is not a significant improvement. It is known that oxygen vacancy is one of the primary causes of fatigue behaviour, thus the observed fatigue characteristics in PLBZT seem to be closely related to the reduced concentration of oxygen vacancies which is less than that in PLLZT.

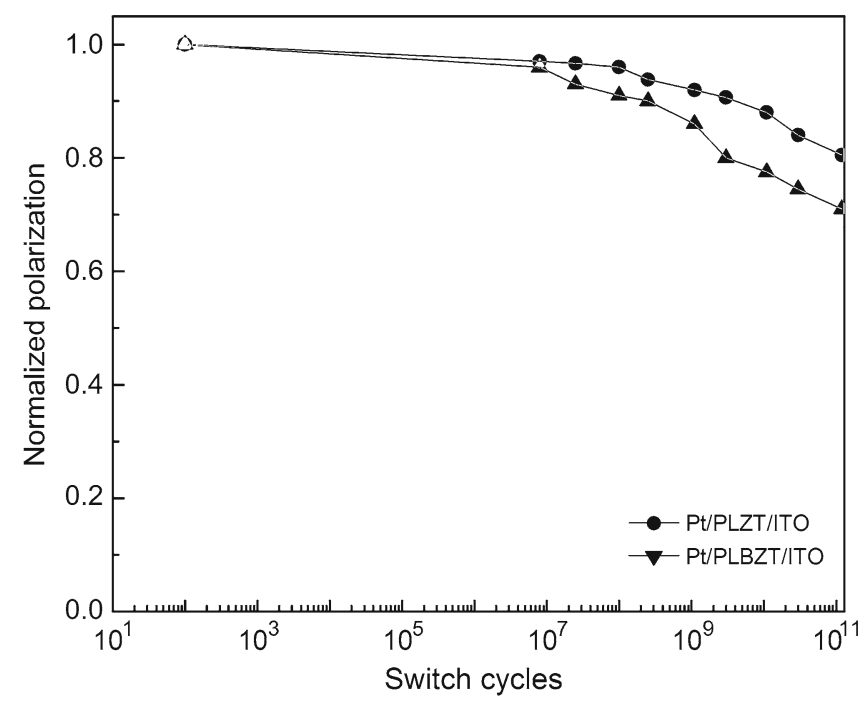

Figure 5. Decay in normalized polarization of PLZT and PLBZT (Bi-doping content, $y=0 \cdot 6$ ) thin films as a function of switching cycles.

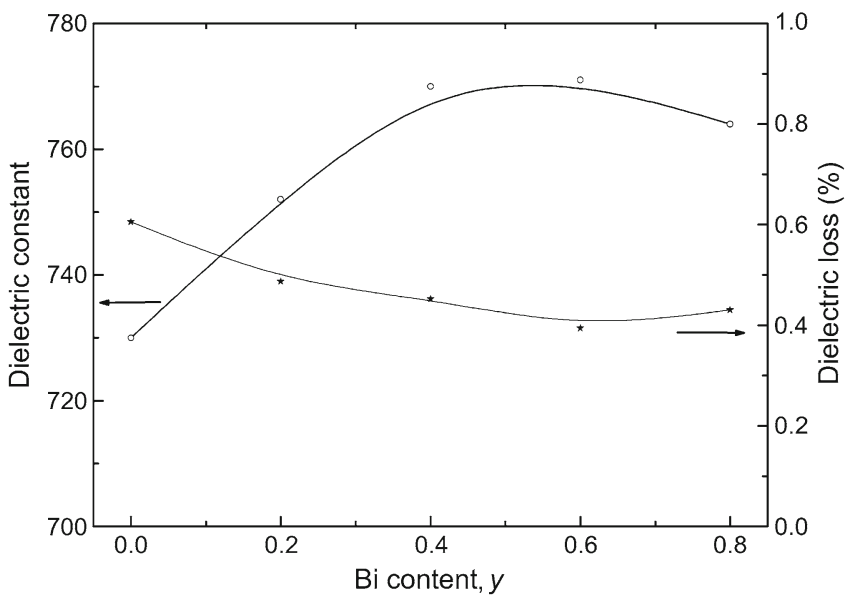

Figure 6. Dielectric constant and dielectric loss of $\left[\mathrm{Pb}_{0.95}\left(\mathrm{La}_{1-y} \mathrm{Bi}_{y}\right)_{0.05}\right]\left[\mathrm{Zr}_{0.53} \mathrm{Ti}_{0.47}\right] \mathrm{O}_{3}$ thin films with various Bi-doping contents, $y$.
Figure 6 shows dielectric constant and dielectric loss of PLBZT films with various Bi-doping contents at $200 \mathrm{kHz}$. From figure 6 , it can be seen that the dielectric constant, $\varepsilon_{\mathrm{r}}$, of PLBZT films first increases, then decreases with the increase in Bi-doping content. When the Bi-doping content $y$ is $0.4, \varepsilon_{\mathrm{r}}$ reaches the largest value of about 770 , which is larger than that of PLZT ( $y=0)$ thin films without Bi-doping. The varying trend in dielectric loss $\tan \delta$ of PLBZT is in contrast to the dielectric constant, $\varepsilon_{\mathrm{r}}$. It has been established that $\varepsilon_{\mathrm{r}}$ decreases with decreasing grain size, the increase in $\varepsilon_{\mathrm{r}}$ and decrease in $\tan \delta$ of PLBZT; with the increase in Bi-doping content from 0.2 to 0.6 is mainly ascribed to the increasing grain size observed in figure 2. However, the structural disorder which is derived from the super-abundant $\mathrm{Bi}$ doping will result in decrease in $\varepsilon_{\mathrm{r}}$ and increase in $\tan \delta$.

A noticeable improvement in leakage current can be confirmed from figure 7. It can be seen that the leakage currents in all samples of PLBZT thin films are lower than $10^{-8} \AA$ at a voltage of $5 \mathrm{~V}$ and these leakage currents are all very much smaller than that of PLZT thin films without $\mathrm{Bi}$. The lowest leakage current value of $2.3 \times 10^{-9} \AA$ can be observed in PLBZT thin films when the Bi-doping content is 0.4 . Nevertheless, as the Bi-doping content is more than 0.4 , leakage current in PLBZT will increase slightly again, in which the varying trend is in contrast to that of dielectric constant for PLBZT thin films. This increase in leakage current may be related to the existence of the pyrochlore phase, which will increase the interface states and defect concentration. Besides, it can be seen that leakage current characteristics of PLBZT films at positive electric field and negative electric field regions are similar as shown in the inset curve in figure 7 . There are two regions of linear increase and exponential increase. The linear increase of leakage current at lower voltage implies that the conductive

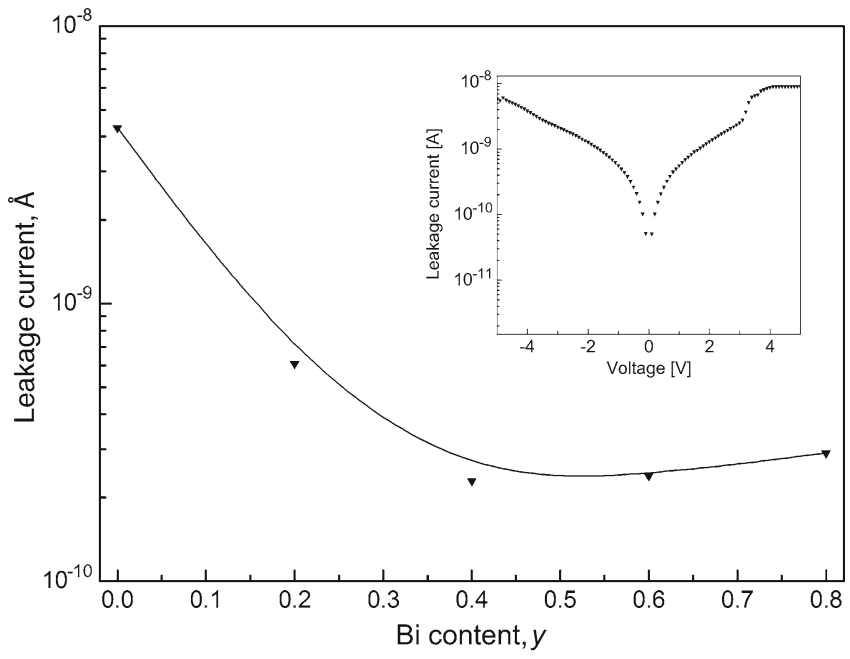

Figure 7. Leakage currents of $\left[\mathrm{Pb}_{0.95}\left(\mathrm{La}_{1-y} \mathrm{Bi}_{y}\right)_{0.05}\right]\left[\mathrm{Zr}_{0.53}\right.$ $\left.\mathrm{Ti}_{0.47}\right] \mathrm{O}_{3}$ thin films and $I-V$ curve of PLBZT with Bidoping content, $y=0 \cdot 4$. 
mechanism is Ohm conduction and the exponential increase of leakage current at higher voltage suggests that the conductive mechanism is Schottky emission. However, slight asymmetry of the $I-V$ characteristic may be attributed to the difference between Pt top electrode and ITO bottom electrode.

\section{Conclusions}

The Bi-doping of PLBZT thin films can be synthesized by sol-gel processing at a relatively low annealing temperature of $550{ }^{\circ} \mathrm{C}$ in oxygen ambient. Bi-doping is useful in crystallization of PLBZT films and promoting grain growth. However, when the Bi-doping content is more than 0.6, the pyrochlore phase appears and the remnant polarization, $P_{\mathrm{r}}$, of PLBZT thin films is smaller than that of PLZT thin films without Bi doping. $P_{\mathrm{r}}$ and $E_{\mathrm{c}}$ of PLBZT thin films are strongly dependent on the Bi-doping content, $y, P_{\mathrm{r}}$ retains almost the same value of $25 \mu \mathrm{C} / \mathrm{cm}^{2}$ with the increase in Bi-doping content from 0.2 to 0.4 , then decreases drastically. PLBZT thin films with excessive Bi-doping content are easier to fatigue than PLZT thin films. When the Bi-doping content, $y$, is not more than 0.4 , dielectric properties, $\varepsilon_{\mathrm{r}}$, decrease to the largest value of about 770 with increase in $\mathrm{Bi}$ doping content, which is larger than that of PLZT thin films without Bi-doping. The lowest leakage current value of $2 \cdot 3 \times$ $10^{-9} \AA$ can be observed in PLBZT thin films when the Bidoping content is 0.4 , which is much smaller than that of PLZT thin films without Bi doping.

\section{Acknowledgements}

This work was supported by the Science Foundation of Guangxi (Grant No. 0832247) and the Guangxi Specific Project Construction of Infrastructure Platform for Science and Technology (Grant No. 10-046-13).

\section{References}

Choudhary R N P and Mal J 2002 Mater. Sci. Eng. B90 1

Gaidi M, Amassian A, Chaker M, Kulishov M and Martinu L 2004 Appl. Surf. Sci. 226347

Goel P and Yadav K L 2007 J. Mater. Sci. 423928

Goel P, Yadav K L and James A R 2004 J. Phys. D: Appl. Phys. 37 3174

Khodorov A and Gomes M J M 2006 Thin Solid Films 5151782

Leclerc G, Domenges B, Poullain G and Bouregba R 2006 Appl. Surf. Sci. 2531143

Mal J and Choudhary R N P 1997 J. Phys. Chem. Solids 58421

Pak J, Park S, Nam K and Park G 2010 Thin Solid Films 5185642

Scott J F 2005 Mater. Sci. Eng. B120 6

Shannigrahi S R, Choudhary R N P, Acharya H N and Sinha T P 1999 J. Appl. Phys. 851713

Shannigrahi S R, Lee S H and Jang H M 2002 J. Am. Ceram. Soc. 852122

Singh R, Goel T C and Chandra S 2008 Mater. Res. Bull. 43384

Tang M H, Zhou Y C, Zheng X J, Yan Z, Cheng C P, Ye Z and Hu Z S 2007 Solid State Electron. 51371

Yoon J E, Cha W H, Lee I S, Kim S J and Son Y G 2008 Surf. Coat. Technol. 203638

Zheng F G, Chen J P, Li X W and Shen M R 2005 Mater. Lett. 59 3498 